\title{
Los ambientes virtuales de aprendizaje: un camino para la educación superior de las personas con alguna necesidad especial de aprendizaje
}

Nancy Edith Ochoa Guevara ${ }^{1}$

\begin{abstract}
Resumen
La alta simulación computacional ha permitido crear ambientes virtuales para que un individuo pueda interactuar en ellos y sentir la realidad como si ocurriera en un entorno verdadero. Este artículo pretende reflexionar sobre la realidad latente de la situación en la formación académica de Educación Superior de la población con alguna necesidad especial de aprendizaje (ciegos, sordos, baja visión, poca audición, pérdida de movilidad física, limitaciones cognitivas, síndrome de Down, autismo, baja estatura y muchas otras formas de discapacidad), que está participando en ambientes virtuales (instructores, estudiantes). Así mismo se analiza el diseño, estructura, esquema y aspectos éticos que debería tener un campus virtual de aprendizaje accesible para esta población en particular. Finalmente, con los aportes de algunos resultados de investigaciones en el tema de los ambientes virtuales, se analizará qué aspectos del diseño del ambiente virtual se deben tener presentes para atender a la población con alguna discapacidad intelectual o física.
\end{abstract}

Palabras clave: accesibilidad, contenido, educación, visual, inclusión, limitación.

1 Ingeniero de Sistemas, Universidad INCCA de Colombia. Especialista en Diseño y Soluciones Telemáticas. Universidad Autónoma de Colombia. MSc (E) Education on line Virtual, Universidad Nacional Abierta y a Distancia, UNAD Florida. 


\title{
Virtual learning environments: a path to higher education for people with special learning needs. social and educational inclusion
}

\begin{abstract}
The high computational simulation has allowed to create virtual atmospheres so that an individual can interact with them and to feel the reality as if it happened in a true environment. This article pretends reflecting about the latent reality of the situation in the academic training of Higher Education, and population with some special learning needs (blind, deaf, limited vision, those of poor hearing, those who have lost physical mobility, those with cognitive limitations, Down syndrome, autism, short stature and many other forms of disability), which are participating in these virtual atmospheres (instructors, students). Also, for this particular population, the design, structure, scheme and ethical aspects that an accessible learning virtual campus should have will be analized, in order to attend people with some physical or intellectual disability.
\end{abstract}

Key words: accessibility, content, education, visual, inclusion, limitation.

\section{Introducción}

La incorporación de las TIC en la educación ha abierto grandes caminos hacia las competencias pedagógicas, tecnológicas y digitales en los ambientes de aprendizaje como Moodle, Claroline, Blackboard, entre otros, pero a la vez ha generado muchas limitaciones a las personas con necesidades especiales de aprendizaje (ciegos, sordos, problemas físicos o mentales).

Es por esto que debemos reflexionar y preguntarnos: ¿ será que hay equidad en los medios y mediaciones?, ¿ por qué las personas con algunas limitaciones físicas y mentales no pueden ingresar en forma independiente a sus aulas virtuales?, En 
este caso, ¿cómo es el aprendizaje para las personas con alguna limitación física o mental en sus aulas virtuales? Estos y muchos más interrogantes nos llevan a reflexionar sobre la urgencia de adaptar los campus virtuales en su diseño instruccional para que sean accesibles a este tipo de población sin cambiar o alterar en su esquema el respectivo modelo pedagógico.

Actualmente, por cada cien (100) estudiantes matriculados en los campus virtuales, aproximadamente el $8 \%$ presenta una discapacidad física o mental y el $7 \%$ no ha manejado un computador y no tiene nociones de internet.

Se debe pensar en una educación inclusiva que abarque todo el sistema educativo y contemple todas las particularidades del entorno a nivel económico, cultural, artístico, político, religioso y laboral. El discapacitado es una persona como las demás, que requiere de un reconocimiento y manejo apropiado de sus condiciones. "La educación es para todos" es un lema que identifica un derecho inalienable de todas las personas, sin mirar su situación física, mental, económica, social, racial, religiosa, cultural o de género.

Actualmente, para la mayoría de las personas del común, ingresar a internet y en especial a una página web puede ser un asunto algo complejo. Esto implica buscar el explorador, conocer el buscador o browser, saber la dirección de la página a donde desea ingresar y tener bien claro qué va a buscar allí entre muchos aspectos. ¿Qué tan complejo puede ser esto para una persona con alguna discapacidad intelectual?, ¿cuántos ensayos y frustraciones podría acarrearle esta actividad? Y es allí donde aparecen los ambientes virtuales capaces de brindarle la oportunidad para analizar, realizar acciones repetitivas y perfeccionarlas hasta obtener el aprendizaje que necesita.

En colaboración con el CERN, Tim Berners-Lee funda en 1994 el Consorcio World Wide Web (W3C) como un consorcio dedicado a generar consenso en relación con las tecnologías web y como opción de normalización ante el creciente desorden de la World Wide Web. Se trata de una asociación internacional formada por organizaciones miembros del consorcio, personal y público en general, que trabajan conjuntamente para desarrollar estándares web. Su misión: guiar la Web hacia su máximo potencial a través del desarrollo de protocolos y pautas que aseguren el crecimiento futuro de la Web. El W3C crea estándares web y pautas para alcanzar su objetivo. En sus primeros diez años ha publicado más de ochenta estándares, como por ejemplo las Recomendaciones. Su trabajo se basa en el desarrollo de tareas de educación y difusión y de software, ofreciendo a su vez un foro abierto para hablar sobre la Web (Gutiérrez, 2001). Para que la Web alcance su máximo potencial, las tecnologías web deben ser compatibles entre sí y permitir que cualquier hardware $o$ software utilizado para acceder a la Web pueda funcionar conjuntamente. El W3C hace referencia a este objetivo denominándolo "interoperabilidad web". Al publicar estándares abiertos (no propietarios) para lenguajes web y protocolos, el W3C busca evitar la fragmentación del mercado y, por lo tanto, de la Web. 
Más de diez años han pasado ya desde que comenzaran las primeras discusiones acerca de la utilización que tendrían los ambientes virtuales (AV) en la accesibilidad de contenidos (diseño de páginas web, preparado para que toda la población pueda navegar, incluyendo las personas con alguna discapacidad, valiéndose de objetos, imágenes, textos, lectores de pantalla y otras herramientas tecnológicas especializadas). Tales discusiones surgieron en gran parte como respuesta a las críticas realizadas respecto a la poca, por no decir ninguna, accesibilidad a los contenidos de las páginas web, pues las herramientas (especializadas para discapacitados) no funcionaban. La rápida evolución de los navegadores, servidores web y demás aplicaciones permite el desarrollo de los ambientes virtuales, aunque en muchas ocasiones se realiza sin tener en cuenta los estándares, lo que trae como consecuencia que los nuevos implementos no puedan ser visualizados o lo sean de forma incorrecta en todas las computadoras. Así surge uno de los grandes problemas de la web actual: la inaccesibilidad.

De este modo, se generó un gran interés en diseñar ambientes virtuales para el entrenamiento y evaluación de destrezas cotidianas en personas con discapacidad intelectual y física, lo cual se tradujo en un aumento de investigaciones sobre el tema. Si bien escasas aún, estas investigaciones han reportado resultados interesantes que hacen necesario un análisis respecto a cuáles son los aportes que los ambientes virtuales podrían hacer en esta área, y bajo qué condiciones dichas aplicaciones serían efectivas. Lo anterior contribuiría al desarrollo del campo y avalaría su utilización en este tipo de población, que por sus características de vulnerabilidad debe estar protegida de potenciales daños derivados de tratamientos novedosos (Aguilar G., 2004).

\section{Ambientes virtuales de aprendizaje}

Existen tres condiciones esenciales para que un ambiente virtual cumpla sus objetivos fundamentales:

- La simulación: Thomas T. Goldsmith Jr. y Estle Ray Mann (1997) la definen así: "Simulación es una técnica numérica para conducir experimentos en una computadora digital. Estos experimentos comprenden ciertos tipos de relaciones matemáticas y lógicas, las cuales son necesarias para describir el comportamiento y la estructura de sistemas complejos del mundo real a través de largos períodos."

- La interacción: Acción de socializar ideas y compartir puntos de vista, conocimientos y posturas con respecto a un objeto de estudios. Esto sólo se da entre personas porque implica una influencia recíproca. 
- La percepción: Es un proceso nervioso superior que permite al organismo, a través de los sentidos, recibir, elaborar e interpretar la información proveniente de su entorno.

Las tres condiciones anteriores permiten que las personas que navegan por los ambientes virtuales con simulación computacional, interactiva y tridimensional sientan que están inmersos en un mundo real gracias a la estimulación brindada por los órganos sensoriales. (Parra et al., 2001).

\section{Las metodologías de trabajo}

\section{a) Tecnología web al alcance de todos}

Existe un amplio debate acerca de la accesibilidad y la inaccesibilidad en la Web. A nivel mundial las posibilidades de acceso están desigualmente distribuidas en las diferentes regiones y países y dependen de un sinnúmero de factores, entre otros: electrificación, extensión de las redes telefónicas en sus diversas formas, disponibilidad de computadores, conectividad, etc. El W3C es parte de la lucha, precisamente, por una Web al alcance de todos. El W3C amplía su campo de influencia a través de la difusión de las tecnologías web que hacen posible que sus beneficios lleguen a los países subdesarrollados. El trabajo realizado en áreas como la accesibilidad web, la internacionalización y la independencia de dispositivo, es especialmente importante en la labor que realiza el W3C por alcanzar una Web para todos.

En el área iberoamericana debemos destacar el trabajo que realiza el SIDAR (Seminario Iberoamericano sobre Discapacidad y Accesibilidad en la Red), en la difusión de los estándares y en la accesibilidad, así como en las traducciones de las Recomendaciones y Pautas del W3C.

\section{b) Web estándar}

Un sitio web construido por estándares web debe tener las siguientes características: formato CSS, texto limpio, imágenes descriptivas, imágenes clasificadas y colores adecuados. Este sitio busca que mediante un lector de pantalla se pueda navegar sin ningún tipo de inconveniente, logrando la accesibilidad, usabilidad y amigabilidad para el usuario y los motores de búsqueda. Analizar con detalle si es necesario el uso de elementos hacks en el CSS para lograr una presentación adecuada, es otro de los pasos a seguir en nuestro camino a una Web estándar. Recordemos que es posible que en futuras versiones de los navegadores éstos no puedan funcionar de forma adecuada si llegan a ocurrir diferenciaciones de uso tecnológico entre desarrolladores y usuarios, lo cual traería como consecuencia una incorrecta visualización de la información y por lo tanto haría inaccesible (Marrero, 2005). 


\section{c) Código correctamente estructurado}

Hacer una perfecta DTD (declaración del tipo de documento) al inicio de nuestros documentos es vital para lograr una internacionalización de nuestra web. Recordemos que la web es el principal medio para difundir la información a nivel regional y global. Por tanto, la web ha de funcionar bajo cualquier circunstancia, en cualquier país, en cualquier idioma y cultura. Una buena internacionalización permite garantizar su uso universal incluyendo todos los idiomas y culturas. Otro de los aspectos a tener en cuenta es revisar nuestro código, usando un validador de (X)HTML y un revisor de CSS, garantizando así la revisión de los mismos por un sistema creado por el W3C con este fin. Tener un código válido significa tener un porcentaje de seguridad de que nuestra web es accesible. Usualmente los diseñadores tienen la costumbre de trabajar con tablas para maquetas. Esto es erróneo ya que ellas se crearon para tabular y presentar estadísticas o datos similares y no para construir un sitio web. Por ejemplo, cuando el afamado Google penetra en un sitio para indexar su contenido, deja para el final la información que está dentro de las tablas.

Por último, y no por ello menos importante, debemos estructurar correctamente nuestro código. Todo debe ubicarse en un orden semántico, por ejemplo, el uso de los encabezados (H1, H2, H3, etc.) anidados correctamente; no utilizar un H3 en un nivel superior al H1; tampoco abusar de elementos DIV, CLASS o ID. Si tenemos una lista de productos, por ejemplo, debemos utilizar las etiquetas que han sido establecidas para ello como UL, OL, DT, etc.

Algunos diseñadores plantean que maquetar un sitio siguiendo las pautas del W3C les crea una limitante a la hora de diseñar y que todos los sitios realizados con la misma se parecen. Sin embargo, parecería que quienes así lo manifiestan no conocen a profundidad esas tecnologías. Existe una variedad de sitios que son verdaderos jardines de diseño empleando solamente (X) HTML y CSS. Entre los más destacados tenemos Csszengarden y Cssbeauty.

\section{d) Accesibilidad}

La accesibilidad en la Web es el acceso universal a los recursos disponibles en ella, independiente del tipo de hardware, software, infraestructura de red, idioma, cultura, localización geográfica y capacidades de los usuarios (Camacho, 1989). El W3C realiza una labor educativa y de concientización en relación con la importancia del diseño accesible de páginas web, uno de los más graves problemas de la Web actual. Hay que tener en cuenta que un sitio accesible debe serlo tanto para las personas sin ningún tipo de dificultad como para aquellos con discapacidad física o intelectual. Tampoco debemos olvidar que el usuario puede estar desenvolviéndose bajo circunstancias externas difíciles como ruidos, demasiada o poca iluminación, entre otros aspectos que afectan la accesibilidad a la información. 


\section{e) Pautas de accesibilidad de la W3C}

Por último, el $\mathrm{W} 3 \mathrm{C}$ ha desarrollado las denominadas Pautas de Accesibilidad al Contenido en la Web, WCAG por sus siglas en inglés. Son en total catorce pautas, cuya función primordial es guiar el diseño de páginas web para hacerlo accesible, reduciendo de esta forma las barreras de la información. Dichas pautas proporcionan soluciones de diseño y ejemplos de cómo una página puede ser inaccesible. Para alcanzar este objetivo, el W3C ha creado puntos de verificación con sus correspondientes niveles de conformidad para lograr que los diferentes grupos de usuarios accedan a la información del sitio Web sin dificultades (Camacho, 2003).

Las pautas también describen cómo hacer páginas web accesibles sin sacrificar el diseño. En este aspecto se repite el debate de si el W3C establece límites a la creatividad del autor en cuanto a diseño. Por el contrario, seguir las pautas ofrece una flexibilidad difícil de alcanzar por otros medios, a la vez que lleva la información a mayor número de usuarios, lo que se traduce en mayor posicionamiento, visibilidad, etc.

\section{f) Usabilidad}

La usabilidad es un aspecto indisoluble de la semántica de la Web. Es la facilidad de uso, ya sea de una página web, de una aplicación informática o de cualquier otro sistema que interactúe con un usuario. Uno de los aspectos ineludibles es que el usuario sepa en todo momento en qué punto de nuestra web se encuentra, mediante una técnica conocida como breadcrumbs o migas de pan. Una adecuada jerarquía visual logra ubicar y estructurar correctamente los elementos de navegación de izquierda a derecha, forma de leer en Occidente. Para distinguir títulos en etiquetas, los conocidos $\mathrm{H} 1, \mathrm{H} 2, \mathrm{H} 3$, no deben faltar en una web estándar, y el uso de los vínculos subrayados es básico para lograr la facilidad de uso de una web. Los enlaces que se han visitado deben ser de color diferente, y para ello tenemos el elemento CSS a:visited. Si preferimos, no establecemos ningún valor para este elemento y lo dejamos en predeterminado. El uso del mapa del sitio es una herramienta verdaderamente útil ya que nos da una visión global del mismo, además de ayudar al usuario a encontrar rápidamente lo que está buscando o informarle en qué lugar se encuentra si se ha perdido.

\section{Análisis y evaluación de algunos resultados de investigación en el tema de los ambientes virtuales para la población con alguna discapacidad intelectual o física}

La crisis de la Educación Superior no es sólo por el tema académico. La crisis abarca también los temas de pertinencia de la oferta educativa y sobre todo de 
equidad en el acceso y de permanencia en las Instituciones de Educación Superior. Éstas a pesar de contar con Sistemas de Autoevaluación y Acreditación de Alta Calidad supervigilados por el Ministerio de Educación Nacional (Sala SACES) y el Comité Nacional de Acreditación (CNA), presentan todavía mucha debilidad en el tema de inclusión educativa, dando la impresión de que es un tema aislado que no compete a la calidad del servicio ni al lema del Ministerio, "revolución educativa" y de algunas instituciones de Educación Superior (IES), "educación para todos". Debemos pensar en la cultura de la calidad y equidad en la Educación Superior, a través de una serie de buenas prácticas, variables e indicadores que pudieran ser incluidos como referentes en la búsqueda de esta cultura de calidad (Wright, B.1988).

a) Investigación: Interculturalidad en las Universidades. Universidad Distrital "Francisco José de Caldas". 2006

En medio de todo el proceso de investigación sobre el tema de la inclusión social y educativa, cito al Dr. Fidel Tubino: "Las políticas de reconocimiento del multiculturalismo son de acción afirmativa y mediante ellas se busca disminuir a corto plazo las grandes asimetrías de oportunidades para permitirle a los grupos vulnerables mejores niveles de igualdad en el acceso al mundo laboral, las representaciones políticas y la educación superior. Funcionan mediante el establecimiento de cuotas teóricamente temporales pero prácticamente crónicas porque no atacan las causas, buscan sólo remediar las consecuencias. Solo logra crear sociedades paralelas o fragmentadas". Esta referencia cierta preocupa y debe ser tomada en cuenta en el momento de analizar las experiencias de acción afirmativa desarrolladas en América Latina.

A continuación se presentan algunos aspectos que se deben tener presentes para lograr avances significativos en la interculturalidad:

1. Que la oferta académica sea pertinente y permita que la comunidad de egresados ejerza su profesión en función del servicio al desarrollo de la región.

2. Que las instituciones estén estrechamente relacionadas con toda la comunidad sin importar su situación física, mental, económica, política, social, cultural y artística, entre otras, lo que permitirá adaptar su oferta académica a las necesidades urgentes de dicha comunidad.

3. Los exámenes de admisión no deben ser de selección, sino al contrario de carácter diagnóstico, para asegurar el apoyo permanente del estudiante durante su formación académica.

La autonomía mal entendida ha impedido la constitución de un espacio común de educación superior, que le otorgue a sus estudiantes coherencia y permanencia en su labor... Zenón Depaz.(2001)(3) 
b) Investigación: La importancia creciente de la accesibilidad de sitios web. Fundación SIDAR. España (2003)

El número de personas que acceden a servicios a través de internet crece cada día. Un porcentaje creciente de estas sufre limitaciones bien sea por su edad avanzada o por algún tipo de discapacidad que le impide realizar sus actividades cotidianas (oír, ver, hablar, valerse por sí mismo). Si al diseñar sitios web nos olvidamos de estas personas, las estamos discriminando. Por otra parte, el acceso a servicios de internet desde contextos y dispositivos variados (TVi, Wireless, PDA), nos obliga a diseñar pensando en facilitar el acceso de este público a la información (Luis Villa, 2003). Estos lineamientos son muy parecidos a los estudios de investigación cuantitativa y cualitativa realizados en la Universidad Jorge Tadeo Lozano y la Universidad Distrital Francisco José de Caldas en Colombia, basados en métodos fenomenológicos hermenéuticos propuestos para la investigación educativa por Spiegelberg y difundidos por Mélich (1997), donde se indaga sobre la discapacidad. Tales investigaciones resaltan que para un buen monitoreo de la inclusión educativa se debe considerar los siguientes aspectos:

1. Iniciar el proceso de selección y aprestamiento en las instituciones desde los primeros periodos académicos.

2. Ofrecer a la población los servicios de Bienestar Universitario con acompañamiento presencial y virtual de los estudiantes de últimos semestres, para relacionarse entre pares, evitar al máximo la marginación y posibilitar el cambio cultural.

3. Hacer énfasis en las carreras de tipo ingenieril o tecnológico que mejoren la calidad de vida en las regiones, buscando el apoyo de profesionales académicos a través de convenios o alianzas.

4. Ubicar a los egresados en sus regiones originarias, valiéndose de charlas y prácticas supervisadas.

c) Investigación: Instituciones de baja calidad (Aguilar G., 2004)

Además de ser la inclusión educativa un tema de poca importancia para la mayoría de Instituciones de Educación Superior, la población discapacitada tiene que lidiar con entes educativos de mala calidad y con un servicio mediocre que lo único que logran es cerrarles el paso en sus intenciones de cultivarse, trayendo como consecuencia el poco interés por continuar con su formación académica y, finalmente, la deserción. Tener en cuenta la accesibilidad a la hora de desarrollar páginas web no es difícil, pero requiere un cambio en la actual forma de trabajar de muchas empresas e instituciones. Por otro lado, tener la accesibilidad en mente desde el inicio es clave, dado que hacer accesible una página web previamente construida es una tarea que puede ser más compleja que crear la página de nuevo. 
Los ambientes virtuales de aprendizaje: un camino para la educación superior de las personas con alguna

necesidad especial de aprendizaje. Inclusión social y educativa, artículo de reflexión

Por eso resulta tentador preguntarse si es un buen negocio hacer una página web accesible.

Analicemos el contexto actual. Se cree que el porcentaje de la población con alguna discapacidad se acerca al $10 \%$. Si consideramos además la creciente longevidad de las personas, no siempre manteniendo las mismas habilidades, nos encontramos con que cada vez más personas con problemas de visión y de movilidad son usuarios habituales de los servicios web. Además, muchas personas pueden estar discapacitadas de manera temporal, por un accidente o una enfermedad, y también en estos casos el uso de Internet puede ser una necesidad que no debe verse frustrada por una mala política de accesibilidad de la web. (Casado, 2006). "Debemos hacer conciencia social sobre el papel que la institución educativa tiene, sobre todo, en cuanto a proporcionar oportunidades equitativas de progreso a todos los miembros de la sociedad, en un marco de respeto hacia las diferencias." (MSc. Ronald Soto Calderón). ${ }^{2}$

d) Investigación: Sistema Dinámico de Información para la población con alguna discapacidad - Proyecto SIDIS. Universidad Nacional Abierta y a Distancia - UNAD. (2009)

El grupo de investigación Sigcienty plantea de forma sucinta una propuesta que pretende orientar acciones hacia la generación de las mejores condiciones para que las entidades del gobierno colombiano dispongan y ejecuten los procesos necesarios para que las herramientas web que generan con destino a todos los ciudadanos colombianos, puedan ser en realidad accedidas por todos en lo que a su navegabilidad y acceso a contenidos se refiere.

El objetivo general del proyecto SIDIS es promover, generar y ejecutar procesos orientados a proporcionar las mejores condiciones para que la Universidad Nacional Abierta y a Distancia, UNAD, en todos los niveles adopte estándares de accesibilidad web que permitan el acceso y navegación de los contenidos de todas sus páginas en el sitio de internet a todo tipo de población sin importar sus condiciones físicas, culturales, políticas, económicas, sociales y artísticas. La UNAD debe implementar mecanismos de formación que favorezcan la incorporación de las condiciones de accesibilidad requeridas para navegación y acceso a los contenidos de los programas que ofrece la Institución (etapa I: limitación visual; etapa II: limitación auditiva; etapa III: limitación física). Teniendo en cuenta que el tema de la accesibilidad web es muy novedoso en nuestro medio, surge como prioridad la implementación de mecanismos de formación a que pueda acceder la comunidad académica y el web máster de la Universidad encargado de incorporar las condiciones en los sitios web. Los

2 Magister en Educación Especial -ICSE-Universidad Pontificia de Comillas, Madrid, España; Magister en Evaluación Educativa de la Universidad de Costa Rica; Sub-director y profesor de la Escuela de Orientación y Educación Especial de la Universidad de Costa Rica; Profesor de la División de Educación Básica del Centro de Investigación y Docencia en Educación de la Universidad Nacional. Profesor de la Maestría en Estudios Interdisciplinarios sobre Discapacidad; Investigador del Instituto de Investigación para el Mejoramiento de la Educación Costarricense (IIMEC). E-mail: ronaldsc@cariari.ucr.ac.cr. 
mecanismos propuestos hacen referencia, de un lado, a la formación virtual y de otro, a estrategias tradicionales. La primera estrategia pretende cubrir en una sola acometida todo el territorio nacional y la segunda, que sectores y regiones que deseen hacer uso de la formación tradicional, tengan esa posibilidad. http://sidis. unad.edu.co.

\section{¿Qué investigar en la inclusión educativa para las personas con alguna discapacidad?}

Más que presentar una estadística de estudiantes con algún tipo de discapacidad o un listado de anomalías o personas con limitaciones, se necesita saber cómo y por qué en la vida cotidiana se excluye a quienes viven con alguna discapacidad casi en todos los sectores sociales, laborales, económicos, culturales y artísticos, siendo un tipo de población plenamente capaz de desempeñar cualquier labor sin ninguna clase de "limitaciones", como se cree. Se peca por ignorancia al cerrarle las puertas a la única opción que tienen de mejorar su calidad de vida a través de su formación académica.

Sea este el medio para invitar a dejar atrás la arrogancia y el rechazo a esta población y aportar en la gestión del conocimiento para enlazar hechos que permitan mirar en forma incluyente a quien vive con exclusión. Por lo tanto debemos preguntarnos:

1. ¿Existen aulas virtuales para la población con necesidades especiales de aprendizaje?

2. ¿En las aulas virtuales existe material accesible para la población discapacitada?

3. ¿Se dispone de un manual o curso de entrenamiento para la población discapacitada en el ambiente virtual?

4. ¿Qué pautas o reglas se deben seguir para atender la población discapacitada en el aula virtual?, ¿Las conoce el instructor y los demás participantes del aula?

Realmente hay una larga serie de aspectos que obligan a reflexionar sobre lo que debemos conocer e indagar en los ambientes virtuales para atender a la población con necesidades especiales, sin tener que modificar al cien por ciento los currículos y las actividades señaladas en las aulas virtuales. 


\section{¿Cuál es la situación de los campus de aprendizaje virtual para las personas con alguna discapacidad y cómo deben ser?}

Algunos proyectos de investigación han arrojado cifras alarmantes sobre la estructura y esquema de ciertos campus virtuales de aprendizaje que impiden la inclusión educativa para las personas con alguna discapacidad por carecer de herramientas de diseño y soporte como Moodle, Blackboard y Claroline, aunque esta última trae un porcentaje de accesibilidad.

Las personas con alguna discapacidad encuentran ciertas dificultades para acceder a sus aulas virtuales, a su e-mail o a un sitio web en particular. La mayoría de las universidades públicas y privadas no están todavía preparadas para atender al cien por ciento este tipo de población, lo que ocasiona un gran traumatismo en el desarrollo de su aprendizaje autónomo, ya que siempre estarán necesitando un tercero para la navegación y exploración en su aula virtual.

La información que circula por la web constituye hoy en día gran parte de la que se maneja en los ámbitos laboral, educativo, cultural e incluso recreativo. Con base en la Constitución Nacional que consigna, de un lado, el derecho al acceso a la información, y de otro, el derecho a la igualdad, se deduce que todos los ciudadanos colombianos cuentan con el derecho de acceso a la información con equidad, aspecto que para las personas en condición de discapacidad es fundamental, ya que se requiere que las herramientas web ofrezcan las condiciones para que todos los usuarios puedan acceder a la información en este entorno, independientemente de sus condiciones físicas, psíquicas o sensoriales (Gutiérrez, 2003).

En el proyecto de investigación SIDIS (Sistema de Información para la Población con Alguna Discapacidad) perteneciente a la Universidad Nacional Abierta y a Distancia, se realizó una encuesta en línea en el año 2008, dirigida a tutores, consejeros académicos, docentes investigadores, administrativos, directivos, estudiantes, egresados y personal externo, la cual arrojó los siguientes resultados:

Tabla 1. Organización de actividades por sesiones

\begin{tabular}{|c|c|c|c|}
\hline No. & Pregunta & Respuesta & $\%$ \\
\hline 1 & $\begin{array}{c}\text { ¿Qué se podría hacer para que } \\
\text { toda la comunidad Unadista se } \\
\text { apropie del tema de la limitación } \\
\text { visual? }\end{array}$ & $\begin{array}{l}\text { Es importante, en primera instancia, realizar } \\
\text { campañas de sensibilización y socialización } \\
\text { acerca del tema, para que de esta manera haya } \\
\text { apropiación y se pueda crear una política interna } \\
\text { de accesibilidad }\end{array}$ & 85 \\
\hline 2 & $\begin{array}{c}\text { ¿Cómo hacer que los } \\
\text { profesionales egresados de } \\
\text { la unad de todas las escuelas } \\
\text { conozcan y apliquen temáticas } \\
\text { de beneficio para la población } \\
\text { con limitación visual? }\end{array}$ & $\begin{array}{l}\text { Generando espacios de participación inclusiva } \\
\text { en el sector laboral y de educación media y } \\
\text { superior, teniendo en cuenta las personas con } \\
\text { limitación visual. }\end{array}$ & 88 \\
\hline
\end{tabular}




\begin{tabular}{|c|c|c|c|}
\hline No. & Pregunta & Respuesta & $\%$ \\
\hline 3 & $\begin{array}{l}\text { ¿Cómo hacer para que el } \\
\text { reconocimiento y la generación } \\
\text { de cursos de accesibilidad web, } \\
\text { tecnología especializada y } \\
\text { conocimientos generales en la } \\
\text { temática de limitación visual, se } \\
\text { aprovechen de la mejor manera } \\
\text { posible? }\end{array}$ & $\begin{array}{c}\text { En cada una de las escuelas se deben } \\
\text { establecer líneas de investigación que relacionen } \\
\text { la inclusión. Bienestar Universitario y Proyección } \\
\text { Social deben organizar jornadas de capacitación } \\
\text { a tutores y docentes en las temáticas } \\
\text { especializadas para que sean multiplicadores de } \\
\text { los conocimientos y los apliquen a la población } \\
\text { objetivo. }\end{array}$ & 90 \\
\hline 4 & $\begin{array}{l}\text { ¿Qué fases o etapas utilizar } \\
\text { para incorporar la temática } \\
\text { de limitación visual en todo el } \\
\text { quehacer universitario? }\end{array}$ & $\begin{array}{l}\text { Desarrollo motor y cognitivo, comunicación y } \\
\text { desarrollo socio-afectivo, aprovechando el PAP } \\
\text { solidario en cada una de las regiones. }\end{array}$ & 85 \\
\hline 5 & $\begin{array}{c}\text { ¿Cómo aprovechar la } \\
\text { temática de limitación visual } \\
\text { para orientar el accionar en } \\
\text { inclusión social hacia las demás } \\
\text { discapacidades? }\end{array}$ & $\begin{array}{l}\text { Desde la función social de las escuelas } \\
\text { existentes en la universidad, desarrollando } \\
\text { planes de trabajo donde se incluya no sólo esta } \\
\text { comunidad sino también los discapacitados en } \\
\text { general. Desarrollando un observatorio para } \\
\text { visibilizar la dinámica y respuesta del programa } \\
\text { para este tipo de población. }\end{array}$ & 92 \\
\hline
\end{tabular}

En la tabla 1 se puede observar el común de las respuestas de cada una de las preguntas, donde se concluye que los estamentos activos de la Universidad Nacional Abierta y a Distancia están totalmente interesados en el tema y a la expectativa de la inclusión educativa tanto en la modalidad tradicional como virtual para la población con alguna Discapacidad. Actualmente ya se han realizado algunos avances significativos en el Centro Regional de Bucaramanga, con el curso académico de inducción unadista para personas sordas (Pinto, 2009).

La accesibilidad en los campus virtuales debe ser con herramientas libres que interactúen conjuntamente aplicando un diseño instruccional que priorice el usuario, la usabilidad del sistema y la accesibilidad de todas las personas con alguna discapacidad. De esta forma, los diversos campus virtuales de diferentes universidades se podrán comunicar e intercambiar mejoras y servicios a nivel nacional e internacional con los participantes. Para conseguirlo, se deben integrar varias plataformas de e-learning en software libre existentes actualmente como Moodle, Claroline y Blackboard, entre otras, contando con la colaboración permanente de Institutos Tecnológicos que apoyen y contribuyan al auge tecnológico sin caer en el "esnobismo" (estar in quedando out por la falta de manejo de la tecnología). Se debe implantar e implementar tecnología en los avances de la educación superior, junto con estándares reconocidos que permitan la inclusión de pautas y reglas en el tema de la accesibilidad en contenidos web.

Estos entornos de campus virtual deben permitir el acceso a personas con discapacidad visual, auditiva, física y de lenguaje, siguiendo los estándares en accesibilidad de la Web Accesibility Initiative (WAI), creados por el reconocido y prestigioso World Wide Web Consortium, de tal manera que se pueda garantizar la accesibilidad de todos los elementos incluidos en el campus para este tipo de población. 


\section{Pautas de accesibilidad}

Las pautas de accesibilidad de la W3C (formato europeo) tienen como objetivo hacer accesibles los contenidos de los sitios web para que la población con alguna discapacidad pueda navegar sin ningún tipo de dificultad. Actualmente Colombia cuenta con unos tres millones de discapacitados: los que tienen baja visión, poca visión, menor movilidad física, limitaciones cognitivas, síndrome de Down, los sordos, ciegos, autistas, los de talla baja y muchas otras formas de discapacidad. Los derechos sólo son humanos si son para todas las personas.

\section{Conclusiones}

En este artículo se ha tratado de dar una visión global de la situación actual de accesibilidad de contenidos en los campus virtuales de aprendizaje para la población con alguna discapacidad, tratando de referenciar los estándares y la accesibilidad en los contenidos Web. Se explican los pasos a seguir para lograr que la información llegue al mayor número de usuarios y sobre todo, para desarrollar un medio donde sea posible el intercambio universal de información, sin ningún tipo de barreras o anomalías.

La inclusión educativa para las personas con alguna discapacidad es necesaria y urgente. Todas las instituciones de Educación Superior deben revisar esta circunstancia, ya que de alguna forma están negándole el derecho de esta población a su formación académica en los niveles superiores.

En los procesos de autoevaluación y acreditación de alta calidad se debe incorporar el tema de la inclusión educativa para las personas con alguna discapacidad. Debe obligarse a los entes de educación superior a tener en cuenta el tema y a aplicarlo en sus mallas curriculares con estrategias metodológicas virtuales, buscando así la equidad en los medios y mediaciones tecnológicas.

No podemos ser ajenos a la situación real de la inclusión educativa para la población con alguna discapacidad, que cada vez está más marginada y, en algunos casos, desmotivada para continuar su formación académica, cerrándose la posibilidad de tener una mejor calidad de vida.

Son el gobierno, la sociedad y las instituciones de educación superior los que deben estar al frente de esta situación. A través de políticas públicas y privadas deben profundizar en el tema de la inclusión educativa en los campus de aprendizaje virtual para las personas con alguna discapacidad. 


\section{Referencias bibliográficas}

Aguilar, G.(2004). Del exterminio de la educación inclusiva: una visión desde la discapacidad. $V$ Congreso Educativo Internacional: De la educación tradicional a la educación inclusiva. Universidad Interamericana. Puerto Rico.

Camacho Rojas, María Felicia. (1989). Ponencia: Hacia el acceso universal: Experiencias en la búsqueda de accesibilidad. Departamento de Educación y Acción Cultural. Banco Central de Costa Rica.

Depaz Toledo, Zenón (2001). “Las fases del método fenomenológico”. En: Del extraño al cómplice. La educación en la vida cotidiana. Rubí (Barcelona): Anthropos, primera reimpresión 1997. p. 54-65.

Gutiérrez Restrepo, Emmanuelle (2000). V Jornada del Seminario SIDAR. Madrid, España.

Pinto, Miguel (2008). Proyecto de Investigación para la población con alguna necesidad especial de aprendizaje (sordos). Universidad Nacional Abierta y a Distancia, UNAD. Bucaramanga, Colombia.

Ruiz Marrero, Carmelo (2005). Artículo de investigación: Nanotecnología: La amenaza de la tecnología enana. Puerto Rico.

Tubino, Fidel (1994). Internacional de Estudios Interculturales. "Interculturalidad: Un Desafío” (con María Heisse; 1992) y “Los efectos de la globalización en la educación” (con Juan Abugattás).

Villa del Campo, Luis (2006). W3C, Fundamentos Web 2006. Madrid, España.

Wright, B. (1988). Desarrollo de conceptos constructivos de vida a partir de la discapacidad. En: Krueger, D. (compilador). Psicología de la Rehabilitación. Caccavari. 